QUARTERLY OF APPLIED MATHEMATICS

VOLUME LXIX, NUMBER 1

MARCH 2011, PAGES 147-155

S 0033-569X(2011)01206-0

Article electronically published on January 18, 2011

\title{
REMARK ON A REGULARITY CRITERION IN TERMS OF PRESSURE FOR THE NAVIER-STOKES EQUATIONS
}

\author{
BY \\ SADEK GALA \\ Department of Mathematics, University of Mostaganem, Box 227, Mostaganem (27000), Algeria
}

Abstract. In this note we establish a Serrin-type regularity criterion in terms of pressure for Leray weak solutions to the Navier-Stokes equation in $\mathbb{R}^{d}$. It is known that if a Leray weak solution $u$ belongs to

$$
L^{\frac{2}{1-r}}\left((0, T) ; L^{\frac{d}{r}}\right) \quad \text { for some } \quad 0 \leq r \leq 1,
$$

then $u$ is regular. It is proved that if the pressure $p$ associated to a Leray weak solution $u$ belongs to

$$
L^{\frac{2}{2-r}}\left((0, T) ; \dot{\mathcal{M}}_{2, \frac{d}{r}}\left(\mathbb{R}^{d}\right)^{d}\right)
$$

where $\dot{\mathcal{M}}_{2, \frac{d}{r}}\left(\mathbb{R}^{d}\right)$ is the critical Morrey-Campanato space (a definition is given in the text) for $0<r<1$, then the weak solution is actually regular. Since this space $\dot{\mathcal{M}}_{2, \frac{d}{r}}$ is wider than $L^{\frac{d}{r}}$ and $\dot{X}_{r}$, the above regularity criterion (0.2) is an improvement of Zhou's result.

1. Introduction. Consider the Navier-Stokes equations in $\mathbb{R}^{d}$ (in particular for $d \leq$ 4):

$$
\begin{array}{rlrl}
\partial_{t} u+u \cdot \nabla u-\Delta u+\nabla p & =0, & & (x, t) \in \mathbb{R}^{d} \times(0, \infty), \\
\operatorname{div} u & =0, & & (x, t) \in \mathbb{R}^{d} \times(0, \infty), \\
u(x, 0) & =a(x), & x \in \mathbb{R}^{d},
\end{array}
$$

where $u=u(x, t)$ is the velocity field, $p=p(x, t)$ is the scalar pressure and $a(x)$ with $\operatorname{div} a=0$ in the sense of distributions is the initial velocity field. For simplicity, we assume that the external force has a scalar potential and is included into the pressure gradient.

In their famous paper, Leray [14] and Hopf [7] constructed a weak solution $u$ of (1.1) for arbitrary $a \in L^{2}\left(\mathbb{R}^{d}\right)$ with $\nabla \cdot a=0$. The solution is called the Leray-Hopf weak solution. Regularity of such Leray-Hopf weak solutions is one of the most significant open problems in mathematical fluid mechanics (see also a recent paper of Y. Zhou 20], 25]). We note here that there are partial regularity results from Scheffer and from Caffarelli,

Received July 30, 2009.

2000 Mathematics Subject Classification. Primary 35Q30, 35K15, 76D05. 
Kohn and Nirenberg (see [4, 5] and the references therein). On the other hand, the work was pioneered by Serrin [15] and extended and improved by Giga [6], Struwe ([18, [19]) and Zhou 25. Further results can be found in 11, 5], 10, and the references therein.

On the other hand, the regularity of a given weak solution $u$ can be shown under an additional condition. Introducing the class $L^{\gamma}\left((0, T) ; L^{\alpha}\left(\mathbb{R}^{d}\right)\right)$, Serrin $[15$ showed that if we have a Leray-Hopf weak solution $u$ belonging to $L^{\gamma}\left((0, T) ; L^{\alpha}\left(\mathbb{R}^{d}\right)\right)$ with the exponents $\alpha$ and $\gamma$ satisfying $\frac{2}{\gamma}+\frac{d}{\alpha}<1,2<\gamma<\infty, d<\alpha<\infty$, then the solution $u(x, t) \in C^{\infty}\left((0, T) \times \mathbb{R}^{d}\right)$, while the limit case $\frac{2}{\alpha}+\frac{d}{q}=1$ was covered much later by H. Sohr [16] (see also [17]). Here we mean by the weak solution a function $u \in$ $L^{\infty}\left((0, T) ; L_{\sigma}^{2}\right) \cap L^{2}\left((0, T) ; H_{\sigma}^{1}\right)$ satisfying (1.1) in the sense of distributions (Definition 3).

Recently, Benbernou [2] proved that if a Leray-Hopf weak solution $u(x, t)$ satisfies the following condition:

$$
p \in L^{\frac{2}{2-r}}\left((0, T) ; \dot{X}_{r}\left(\mathbb{R}^{d}\right)^{d}\right),
$$

then $u(x, t)$ actually is a strong solution of (1.1) on $(0, T)$.

Regularity results including assumptions on the pressure have been given by Kaniel [8], and more recently a final result has been obtained by Berselli and Galdi [3] (a much simpler proof was given by Zhou 21], very recently). It is shown that if the pressure $p \in L^{\alpha}\left((0, T) ; L^{q}\left(\mathbb{R}^{d}\right)\right)$ with $\frac{2}{\alpha}+\frac{d}{q} \leq 2$, then the corresponding weak solution actually is strong.

The purpose of this work is to establish a Serrin-type regularity criterion in terms of the pressure for weak solutions to the Navier-Stokes equations in the class $L^{\frac{2}{2-r}}((0, T)$; $\left.\dot{\mathcal{M}}_{2, \frac{d}{r}}\left(\mathbb{R}^{d}\right)^{d}\right)$. For more facts concerning regularity of weak solutions, we refer the reader to the celebrated papers of Y. Zhou ([20, [25]).

Now, we recall the definition and some properties of the space that we are going to use. These spaces play an important role in studying the regularity of solutions to partial differential equations; see e.g. [11, 26] and the references therein.

Definition 1. For $0 \leq r<\frac{d}{2}$, the space $\dot{X}_{r}$ is defined as the space of $f(x) \in L_{\text {loc }}^{2}\left(\mathbb{R}^{d}\right)$ such that

$$
\|f\|_{\dot{X}_{r}}=\sup _{\|g\|_{\dot{H}^{r} \leq 1}}\|f g\|_{L^{2}}<\infty
$$

where we denote by $\dot{H}^{r}\left(\mathbb{R}^{d}\right)$ the completion of the space $\mathcal{D}\left(\mathbb{R}^{d}\right)$ with respect to the norm $\|u\|_{\dot{H}^{r}}=\left\|(-\Delta)^{\frac{r}{2}} u\right\|_{L^{2}}$.

We have the homogeneity properties: $\forall x_{0} \in \mathbb{R}^{d}$,

$$
\begin{aligned}
\left\|f\left(.+x_{0}\right)\right\|_{\dot{X}_{r}} & =\|f\|_{\dot{X}_{r}}, \\
\|f(\lambda .)\|_{\dot{X}_{r}} & =\frac{1}{\lambda^{r}}\|f\|_{\dot{X}_{r}}, \quad \lambda>0 .
\end{aligned}
$$

The following imbedding,

$$
L^{\frac{d}{r}}\left(\mathbb{R}^{d}\right) \subset \dot{X}_{r}\left(\mathbb{R}^{d}\right), \quad 0 \leq r<\frac{d}{2}
$$

holds. For the proof, see for example [26]. 
Now we recall the definition of Morrey-Campanato spaces:

Definition 2. For $1<p \leq q \leq+\infty$, the Morrey-Campanato space $\dot{\mathcal{M}}_{p, q}$ is defined by

$$
\dot{\mathcal{M}}_{p, q}=\left\{f \in L_{l o c}^{p}\left(\mathbb{R}^{d}\right):\|f\|_{\mathcal{M}_{p, q}}=\sup _{x \in \mathbb{R}^{d}} \sup _{R>0} R^{d / q-d / p}\|f\|_{L^{p}(B(x, R))}<\infty\right\} .
$$

It is easy to verify that $\dot{\mathcal{M}}_{p, q}\left(\mathbb{R}^{d}\right)$ is a Banach space under the norm $\|\cdot\|_{\dot{\mathcal{M}}_{p, q}}$. Furthermore, it is easy to check the following:

$$
\|f(\lambda .)\|_{\mathcal{M}_{p, q}}=\frac{1}{\lambda^{\frac{d}{q}}}\|f\|_{\mathcal{M}_{p, q}}, \quad \lambda>0 .
$$

We have the following comparison between Lorentz spaces and Morrey-Campanato spaces: for $p \geq 2$,

$$
L^{\frac{d}{r}}\left(\mathbb{R}^{d}\right) \subset L^{\frac{d}{r}, \infty}\left(\mathbb{R}^{d}\right) \subset \dot{\mathcal{M}}_{p, \frac{d}{r}}\left(\mathbb{R}^{d}\right)
$$

The relation

$$
L^{\frac{d}{r}, \infty}\left(\mathbb{R}^{d}\right) \subset \dot{\mathcal{M}}_{p, \frac{d}{r}}\left(\mathbb{R}^{d}\right)
$$

is shown as follows. Let $f \in L^{\frac{d}{r}, \infty}\left(\mathbb{R}^{d}\right)$. Then

$$
\begin{aligned}
\|f\|_{\mathcal{M}_{p, \frac{3}{r}}} & \leq \sup _{E}|E|^{\frac{r}{d}-\frac{1}{2}}\left(\int_{E}|f(y)|^{p} d y\right)^{\frac{1}{p}} \\
& =\left(\sup _{E}|E|^{\frac{p r}{d}-1} \int_{E}|f(y)|^{p} d y\right)^{\frac{1}{p}} \\
& \cong\left(\sup _{R>0} R\left|\left\{x \in \mathbb{R}^{d}:|f(y)|^{p}>R\right\}\right|^{\frac{p r}{d}}\right)^{\frac{1}{p}} \\
& =\sup _{R>0} R\left|\left\{x \in \mathbb{R}^{d}:|f(y)|>R\right\}\right|^{\frac{r}{d}} \\
& \cong\|f\|_{L^{\frac{d}{r}, \infty}} .
\end{aligned}
$$

For $0<r<1$, we use the fact that

$$
L^{2} \cap \dot{H}^{1} \subset \dot{B}_{2,1}^{r} \subset \dot{H}^{r} .
$$

Thus we can replace the space $\dot{X}_{r}$ by the pointwise multipliers from the Besov space $\dot{B}_{2,1}^{r}$ to $L^{2}$. Then we have the following lemma given in [12].

Lemma 1 . For $0 \leq r<\frac{d}{2}$, the space $\dot{Z}_{r}$ is defined as the space of $f(x) \in L_{l o c}^{2}\left(\mathbb{R}^{d}\right)$ such that

$$
\|f\|_{\dot{Z}_{r}}=\sup _{\|g\|_{\dot{B}_{2,1}^{r}} \leq 1}\|f g\|_{L^{2}}<\infty
$$

Then $f \in \dot{\mathcal{M}}_{2, \frac{d}{r}}$ if and only if $f \in \dot{Z}_{r}$ with equivalence of norms.

To prove our main result, we need the following lemma. 
Lemma 2. For $0<r<1$, we have

$$
\|f\|_{\dot{B}_{2,1}^{r}} \leq C\|f\|_{L^{2}}^{1-r}\|\nabla f\|_{L^{2}}^{r} .
$$

Proof 1. The idea comes from [13. According to the definition of Besov spaces, one has

$$
\begin{aligned}
\|f\|_{\dot{B}_{2,1}^{r}} & =\sum_{j \in \mathbb{Z}} 2^{j r}\left\|\Delta_{j} f\right\|_{L^{2}} \\
& \leq \sum_{j \leq k} 2^{j r}\left\|\Delta_{j} f\right\|_{L^{2}}+\sum_{j>k} 2^{j(r-1)} 2^{j}\left\|\Delta_{j} f\right\|_{L^{2}} \\
& \leq\left(\sum_{j \leq k} 2^{2 j r}\right)^{\frac{1}{2}}\left(\sum_{j \leq k}\left\|\Delta_{j} f\right\|_{L^{2}}^{2}\right)^{\frac{1}{2}}+\left(\sum_{j>k} 2^{2 j(r-1)}\right)^{\frac{1}{2}}\left(\sum_{j>k} 2^{2 j}\left\|\Delta_{j} f\right\|_{L^{2}}^{2}\right)^{\frac{1}{2}} \\
& \leq C\left(2^{r k}\|f\|_{L^{2}}+2^{k(r-1)}\|f\|_{\dot{H}^{1}}\right) \\
& =C\left(2^{r k} A^{-r}+2^{k(r-1)} A^{1-r}\right)\|f\|_{L^{2}}^{1-r}\|f\|_{\dot{H}^{1}}^{r},
\end{aligned}
$$

where $A=\frac{\|f\|_{H^{1}}}{\|f\|_{L^{2}}}$. Choosing $k$ such that $2^{r k} A^{-r} \leq 1$, that is, $k \leq\left[\log A^{r}\right]$, we thus obtain

$$
\begin{aligned}
\|f\|_{\dot{B}_{2,1}^{r}} & \leq C\left(1+2^{k(r-1)} A^{1-r}\right)\|f\|_{L^{2}}^{1-r}\|f\|_{\dot{H}^{1}}^{r} \\
& \leq C\|f\|_{L^{2}}^{1-r}\|\nabla f\|_{L^{2}}^{r} .
\end{aligned}
$$

Additionally, for $2<p \leq \frac{d}{r}$ and $0 \leq r<\frac{d}{2}$, we have the following inclusion relations ([11, [12]):

$$
\dot{\mathcal{M}}_{p, \frac{d}{r}}\left(\mathbb{R}^{d}\right) \subset \dot{X}_{r}\left(\mathbb{R}^{d}\right) \subset \dot{\mathcal{M}}_{2, \frac{d}{r}}\left(\mathbb{R}^{d}\right)=\dot{Z}_{r}\left(\mathbb{R}^{d}\right)
$$

The relation

$$
\dot{X}_{r}\left(\mathbb{R}^{d}\right) \subset \dot{\mathcal{M}}_{2, \frac{d}{r}}\left(\mathbb{R}^{d}\right)
$$

is shown as follows. Let $f \in \dot{X}_{r}\left(\mathbb{R}^{d}\right), 0<R \leq 1, x_{0} \in \mathbb{R}^{d}$ and $\phi \in C_{0}^{\infty}\left(\mathbb{R}^{d}\right), \phi \equiv 1$ on $B\left(\frac{x_{0}}{R}, 1\right)$. We have

$$
\begin{aligned}
R^{r-\frac{d}{2}}\left(\int_{\left|x-x_{0}\right| \leq R}|f(x)|^{2} d x\right)^{1 / 2} & =R^{r}\left(\int_{\left|y-\frac{x_{0}}{R}\right| \leq 1}|f(R y)|^{2} d y\right)^{1 / 2} \\
& \leq R^{r}\left(\int_{y \in \mathbb{R}^{d}}|f(R y) \phi(y)|^{2} d y\right)^{1 / 2} \\
& \leq R^{r}\|f(R .)\|_{\dot{X}_{r}}\|\phi\|_{H^{r}} \\
& \leq\|f\|_{\dot{X}_{r}}\|\phi\|_{H^{r}} \\
& \leq C\|f\|_{\dot{X}_{r}} .
\end{aligned}
$$

Since $L^{\frac{d}{r}}\left(\mathbb{R}^{d}\right) \subset \dot{X}_{r}\left(\mathbb{R}^{d}\right) \subset \dot{\mathcal{M}}_{2, \frac{d}{r}}\left(\mathbb{R}^{d}\right)$, the above regularity criterion is an improvement on the Zhou and Benbernou results, and hence our regularity criterion covers the recent result given by [2] and [24]. 
2. Regularity theorem. In this section we give the regularity criterion involving the integrability of the pressure for the Leray-type weak solution of the Navier-Stokes equation (1.1). We start with some prerequisites for our main results. Let

$$
C_{0, \sigma}^{\infty}\left(\mathbb{R}^{d}\right)=\left\{\varphi \in\left(C_{0}^{\infty}\left(\mathbb{R}^{d}\right)\right)^{d}: \operatorname{div} \varphi=0\right\} \subseteq\left(C_{0}^{\infty}\left(\mathbb{R}^{d}\right)\right)^{d} .
$$

The subspace

$$
L_{\sigma}^{2}\left(\mathbb{R}^{d}\right)=\overline{C_{0, \sigma}^{\infty}\left(\mathbb{R}^{d}\right)} \|^{\|\cdot\|_{L^{2}}}=\left\{u \in L^{2}\left(\mathbb{R}^{d}\right)^{d}: \operatorname{div} u=0\right\}
$$

is obtained as the closure of $C_{0, \sigma}^{\infty}$ with respect to the $L^{2}$-norm $\|\cdot\|_{L^{2}} . H_{\sigma}^{r}$ is the closure of $C_{0, \sigma}^{\infty}$ with respect to the $H^{r}$-norm

$$
\|u\|_{H^{r}}=\left\|(1-\Delta)^{\frac{r}{2}} u\right\|_{L^{2}}, \text { for } r \geq 0 .
$$

Next we recall the definition of Leray-Hopf weak solutions (see [14]).

Definition 3 (weak solutions). Let $a \in L_{\sigma}^{2}$ and $T>0$. A measurable function $u$ is called a weak solution of Leray-Hopf type (a Leray-Hopf solution) on $(0, T)$ if $u$ satisfies the following properties:

(1): $u \in L^{\infty}\left((0, T) ; L_{\sigma}^{2}\right) \cap L^{2}\left((0, T) ; H_{\sigma}^{1}\right)$ for all $T>0$;

(2): $u(t)$ is continuous in time in the weak topology of $L_{\sigma}^{2}$ with

$$
\langle u(t), \phi\rangle \rightarrow\langle a, \phi\rangle \quad \text { as } t \rightarrow+0
$$

for all $\phi \in L_{\sigma}^{2}$;

(3): for any $0 \leq s \leq t \leq T, u$ satisfies the identity

$$
\begin{aligned}
& \int_{s}^{t}\left\{-\left\langle u, \partial_{\tau} \phi\right\rangle+\langle u \cdot \nabla u, \phi\rangle+\langle\nabla u, \nabla \phi\rangle\right\} d \tau=-\langle u(t), \phi(t)\rangle+\langle u(s), \phi(s)\rangle, \\
& \quad \text { for all } \phi \in H^{1}\left((s, t) ; H_{\sigma}^{1}\right) .
\end{aligned}
$$

Our result on the regularity criterion for weak solutions now reads as follows.

Theorem 1 (Regularity Criterion). Let $a \in L^{2}\left(\mathbb{R}^{d}\right) \cap L^{q}\left(\mathbb{R}^{d}\right)$ for some $q \geq d$, and $\nabla \cdot a=0$ in the sense of distributions. Suppose that $u(t, x)$ is a Leray-Hopf solution of (1.1) in $[0, T)$. If the pressure $p$ satisfies

$$
\left.\left.p \in L^{\gamma}\left((0, T), \dot{\mathcal{M}}_{2, \frac{d}{r}}\left(\mathbb{R}^{d}\right)\right) \text { with } r \in\right] 0,1\right] \text { and } \gamma=\frac{2}{2-r},
$$

then $u(t, x)$ is a regular solution in the sense that

$$
u \in C^{\infty}\left([0, T] \times \mathbb{R}^{d}\right) .
$$

In order to prove Theorem 1. we recall the well-known pressure-velocity relation in $\mathbb{R}^{d}$, given by

$$
p=\sum_{i, j=1}^{d} \mathcal{R}_{i} \mathcal{R}_{j}\left(u_{i} u_{j}\right),
$$

where $\left(\mathcal{R}_{i}\right)_{i=1}^{d}$ are the Riesz transforms in $\mathbb{R}^{d}$. The Calderón-Zygmund inequality implies then

$$
\|p\|_{\alpha} \leq\|u\|_{2 \alpha}^{2}, \quad 1<\alpha<+\infty
$$


We recall the following result due to Y. Giga [6] (see also [9], [3]) that will be used in the proof of Theorem 1, $B C$ denotes the class of bounded and continuous functions.

Lemma 3. Suppose that $a \in L^{\alpha}\left(\mathbb{R}^{d}\right)^{d}$, for $\alpha \geq d$ and $\nabla \cdot a=0$. Then, there exists $T_{0}>0$ and a unique solution of (1.1) on $\left[0, T_{0}\right)$ such that

$$
\left\{\begin{array}{c}
u \in B C\left(\left[0, T_{0}\right) ; L^{\alpha}\left(\mathbb{R}^{d}\right)^{d}\right) \cap L^{r}\left(\left[0, T_{0}\right) ; L^{s}\left(\mathbb{R}^{d}\right)^{d}\right), \\
t^{\frac{1}{r}} u \in B C\left(\left[0, T_{0}\right) ; L^{s}\left(\mathbb{R}^{d}\right)^{d}\right),
\end{array}\right.
$$

where $\frac{2}{r}+\frac{d}{s}=\frac{d}{q}, s>d$. Moreover, let $\left(0, T^{*}\right)$ be the maximal interval such that $u$ solves (1.1) in $C\left(\left(0, T^{*}\right) ; L^{\alpha}\left(\mathbb{R}^{d}\right)^{d}\right), \alpha>d$. Then for any $t \in\left(0, T^{*}\right)$,

$$
\|u(t)\|_{L^{\alpha}} \geq \frac{C}{\left(T^{*}-t\right)^{\frac{\alpha-d}{2 \alpha}}}
$$

with the constant $C$ independent of $T^{*}$ and $\alpha$.

(c): Let $u$ be a strong solution satisfying

$$
u \in L^{\alpha}\left((0, T) ; L^{\beta}\left(\mathbb{R}^{d}\right)^{d}\right) \quad \text { for } \frac{2}{\alpha}+\frac{d}{\beta}=1 \text { and } \beta>d .
$$

Then $u$ belongs to $C^{\infty}\left(\mathbb{R}^{d} \times(0, T)\right)$.

We make two remarks.

REMARK 1. The existence of weak solutions is generally known but their uniqueness and regularity remain an open problem. The weak solution $u \in L^{\infty}\left((0, T) ; L_{\sigma}^{2}\right) \cap$ $L^{2}\left((0, T) ; \dot{H}_{\sigma}^{1}\right)$ was constructed by Leray as the initial velocity $a \in L_{\sigma}^{2}$, such that $u$ satisfies the energy inequality.

REMARK 2. By a strong solution we mean a weak solution of the Navier-Stokes equation such that

$$
u \in L^{\infty}\left((0, T) ; H^{1}\right) \cap L^{2}\left((0, T) ; H^{2}\right) .
$$

It is well known that strong solutions are regular (we say classical) and unique in the class of weak solutions.

3. Proof of Theorem 1, The proof consists in first obtaining a continuation principle for strong solutions and then in applying it to weak solutions.

Proof. By using the results of the previous Lemma 3, the weak solution $u$ is smooth in some time interval $\left(0, T^{*}\right), T^{*} \leq T$. In particular, $(u, p) \in C^{\infty}\left(\mathbb{R}^{d} \times\left(0, T^{*}\right)\right)$ and $u$ is in the class (2.4). Thus, for any $T>0$ we suppose that $u$ is a smooth solution to (1.1) on $\mathbb{R}^{d} \times(0, T)$ and will establish a priori bounds that will allow us to extend $u$ for all time. Hence, it suffices to establish the following a priori estimate:

$$
\sup _{0 \leq t \leq T}\|u(t)\|_{L^{\alpha}}^{\alpha} \leq\|a\|_{L^{\alpha}}^{\alpha} \exp \left(C \int_{0}^{t}\|p\|_{\mathcal{\mathcal { M }}_{2, \frac{d}{r}}}^{\frac{2}{2-r}} d s\right),
$$

where $C$ is independent of $T$. 
In order to prove our main result, we multiply both sides of (1.1) by $\alpha u|u|^{\alpha-2}$ for some $\alpha \geq d$ : we obtain after suitable integration by parts (see e.g. 24])

$$
\begin{aligned}
& \|u(., t)\|_{L^{\alpha}}^{\alpha}+\alpha\left\||\nabla u||u|^{\frac{\alpha}{2}-1}\right\|_{L^{2,2}}^{2}+\frac{4(\alpha-2)}{\alpha}\left\|\nabla|u|^{\frac{\alpha}{2}}\right\|_{L^{2,2}}^{2} \\
\leq & \left.2(\alpha-2) \int_{0}^{t} \int_{\mathbb{R}^{d}}|p||u|^{\frac{\alpha}{2}-1}|\nabla| u\right|^{\frac{\alpha}{2}} \mid d x d s+\|a\|_{L^{\alpha}}^{\alpha},
\end{aligned}
$$

for $t \in(0, T)$. Let $w=|u|^{\frac{\alpha}{2}}$. Then Cauchy's inequality implies that

$$
\begin{aligned}
& \|u(t)\|_{L^{\alpha}}^{\alpha}+\alpha\left\||\nabla u||u|^{\frac{\alpha}{2}-1}\right\|_{L^{2,2}}^{2}+\frac{4(\alpha-2)}{\alpha}\|\nabla w\|_{L^{2,2}}^{2} \\
\leq & 2(\alpha-2) \int_{0}^{t} \int_{\mathbb{R}^{d}}|p||w|^{1-\frac{2}{\alpha}}|\nabla w| d x d s+\|a\|_{L^{\alpha}}^{\alpha} \\
\leq & (\alpha-2)\left[\int_{0}^{t} \int_{\mathbb{R}^{d}}|\nabla w|^{2} d x d s+\int_{0}^{t} \int_{\mathbb{R}^{d}}|p|^{2}|w|^{2\left(1-\frac{2}{\alpha}\right)} d x d s\right]+\|a\|_{L^{\alpha}}^{\alpha} .
\end{aligned}
$$

Let us estimate the integral

$$
I=\int_{0}^{t} \int_{\mathbb{R}^{d}}|p|^{2}|w|^{2\left(1-\frac{2}{\alpha}\right)} d x d s
$$

on the right-hand side of (3.2). By the Hölder inequality and the Young inequality we have

$$
\begin{aligned}
I & \leq \int_{0}^{t}\|p w\|_{L^{2}}\left\|p w^{\frac{\alpha-4}{\alpha}}\right\|_{L^{2}} d s \\
& \leq \int_{0}^{t}\left(\|p\|_{\mathcal{M}_{2, \frac{d}{r}}}\|w\|_{\dot{B}_{2,1}^{r}}^{r}\right)\left(\|p\|_{L^{\frac{\alpha}{2}}}\left\|w^{\frac{\alpha-4}{\alpha}}\right\|_{L^{\frac{2 \alpha}{\alpha-4}}}\right) d s \\
& \leq C \int_{0}^{t}\|p\|_{\mathcal{\mathcal { M }}_{2, \frac{d}{r}}}\|w\|_{L^{2}}^{1-r}\|\nabla w\|_{L^{2}}^{r}\left\||u|^{2}\right\|_{L^{\frac{\alpha}{2}}}\|w\|_{L^{2}}^{\frac{\alpha-4}{\alpha}} d s \\
& \leq C \int_{0}^{t}\|p\|_{\mathcal{\mathcal { M }}_{2, \frac{d}{r}}}\|w\|_{L^{2}}^{1-r}\|\nabla w\|_{L^{2}}^{r}\|w\|_{L^{2}} d s \\
& \leq C \int_{0}^{t}\left(\|p\|_{\mathcal{M}_{2, \frac{d}{r}}}\|w\|_{L^{2}}^{2-r}\right)\|\nabla w\|_{L^{2}}^{r} d s \\
& \leq \epsilon \int_{0}^{t}\|\nabla w\|_{L^{2}}^{2} d s+C(\epsilon) \int_{0}^{t}\|p\|_{\mathcal{M}_{2, \frac{d}{r}}^{2-r}}^{\frac{2}{2-r}}\|w\|_{L^{2}}^{2} d s,
\end{aligned}
$$

where we used

$$
\|w\|_{\dot{B}_{2,1}^{r}} \leq C\|w\|_{L^{2}}^{1-r}\|\nabla w\|_{L^{2}}^{r}
$$

and

$$
\|p\|_{\alpha} \leq\|u\|_{2 \alpha}^{2}, \quad 1<\alpha<+\infty .
$$

Here we made use of the Young inequality with $\epsilon$ :

$$
a b \leq \epsilon a^{p}+C(\epsilon) b^{q} \quad(a, b>0, \epsilon>0) \quad \text { and } \quad\left(\frac{1}{p}+\frac{1}{q}=1\right),
$$


for $C(\epsilon)=(\epsilon p)^{-\frac{q}{p}} q^{-1}$. Since

$$
\left\||\nabla u||u|^{\frac{\alpha}{2}-1}\right\|_{L^{2}}^{2}=\frac{4}{\alpha^{2}}\left\|\nabla|u|^{\frac{\alpha}{2}}\right\|_{L^{2}}^{2}=\frac{4}{\alpha^{2}}\|\nabla w\|_{L^{2}}^{2},
$$

then by (3.2) and the above equality, we derive

$$
\begin{aligned}
& \|u(t)\|_{L^{\alpha}}^{\alpha}+\frac{4(\alpha-1)}{\alpha}\|\nabla w\|_{L^{2,2}}^{2} \\
\leq & (\alpha-2)(\epsilon+1) \int_{0}^{t}\|\nabla w\|_{L^{2}}^{2} d s+C(\alpha, \epsilon) \int_{0}^{t}\|p\|_{\mathcal{M}_{2, \frac{d}{r}}^{2-r}}^{\frac{2}{2-r}}\|w\|_{L^{2}}^{2} d s+\|a\|_{L^{\alpha}}^{\alpha},
\end{aligned}
$$

that is,

$$
\|u(t)\|_{L^{\alpha}}^{\alpha}+K(\alpha, \epsilon) \int_{0}^{t}\left\|\nabla|u|^{\frac{\alpha}{2}}\right\|_{L^{2}}^{2} d s \leq\|a\|_{L^{\alpha}}^{\alpha}+C(\alpha, \epsilon) \int_{0}^{t}\|p\|_{\mathcal{M}_{2, \frac{d}{r}}}^{\frac{2}{2-r}}\|w\|_{L^{2}}^{2} d s,
$$

where we take $\epsilon$ to be sufficiently small, and then fix it. Due to Gronwall's inequality, it follows from (3.4) that

$$
\sup _{0 \leq t \leq T}\|u(t)\|_{L^{\alpha}}^{\alpha} \leq\|a\|_{L^{\alpha}}^{\alpha} \exp \left(C \int_{0}^{t}\|p\|_{\mathcal{M}_{2, \frac{d}{r}}^{2-r}}^{\frac{2}{r}} d s\right)
$$

and consequently

$$
u \in L^{\infty}\left([0, T), L^{\alpha}\left(\mathbb{R}^{d}\right)^{d}\right) .
$$

This estimate assures that the solution has the regularity in (3.6) under the assumption (2.2) which yields

$$
u \in L^{s}\left([0, T), L^{\alpha}\left(\mathbb{R}^{d}\right)^{d}\right) \quad \text { with } \frac{2}{s}+\frac{d}{\alpha}=1,
$$

from which and Lemma 3 the smoothness of $u$ follows immediately. This completes the proof of Theorem 1

Acknowledgement. The author would like to express his sincere gratitude to Professor Yong Zhou for his useful comments and constant encouragement.

\section{REFERENCES}

[1] H. Beirão da Veiga, A sufficient condition on the pressure for the regularity of weak solutions to the Navier-Stokes equations, J. Math. Fluid Mech. 2 (2000), 99-106. MR1765772 (2001d:76025)

[2] S. Benbernou, A note on the regularity criterion in terms of pressure for the Navier-Stokes equations, Appl. Math. Letters 22 (2009), 1438-1443. MR2536829

[3] L.C. Berselli and G.P. Galdi, Regularity criteria involving the pressure for the weak solutions to the Navier-Stokes equations, Proc. Amer. Math. Soc. 130 (2002), 3585-3595. MR1920038(2003e:35240)

[4] L. Caffarelli, J. Kohn and L. Nirenberg, Partial regularity for suitable weak solutions of the NavierStokes equations, Comm. Pure Appl. Math. 35 (1982), 771-831. MR673830 (84m:35097)

[5] D. Chae and J. Lee, Regularity criterion in terms of pressure for the Navier-Stokes equations, Nonlinear Anal. 46 (2001), 727-735. MR.1857154(2002g:76032)

[6] Y. Giga, Solutions for semilinear parabolic equations in $L^{p}$ and regularity of weak solutions of the Navier-Stokes system, J. Differential Equations 62 (1986), 186-212. MR.833416 (87h:35157)

[7] E. Hopf, Über die Anfangswertaufgabe für die hydrodynamischen Grundgleichungen, Math. Nachr. 4 (1951), 213-231. MR0050423 (14:327b)

[8] S. Kaniel, A sufficient condition for smoothness of solutions of Navier-Stokes equations, Israel J. Math. 6 (1968), 354-358. MR0244651 (39:5965) 
[9] T. Kato, Strong $L^{p}$-solutions of the Navier-Stokes equation in $\mathbb{R}^{m}$, with applications to weak solutions, Math. Z. 187 (1984), 471-480. MR760047 (86b:35171)

[10] H. Kozono and H. Sohr, Regularity criterion on weak solutions to the Navier-Stokes equations, Adv. Differential Equations 2 (1997), 535-554. MR.1441855 (97m:35206)

[11] P.G. Lemarié-Rieusset, Recent Developments in the Navier-Stokes Problem. London: Chapman \& Hall/CRC, 2002. MR.1938147 (2004e:35178)

[12] P.G. Lemarié-Rieusset, The Navier-Stokes equations in the critical Morrey-Campanato space. Rev. Mat. Iberoam. 23 (2007), no. 3, 897-930. MR2414497 (2009g:35221)

[13] S. Machihara and T. Ozawa, Interpolation inequalities in Besov spaces, Proc. Amer. Math. Soc. 131 (2003), 1553-1556. MR 1949885 (2004i:46030)

[14] J. Leray, Sur le mouvement d'un liquide visqueux emplissant l'espace, Acta Math. 63 (1934), 193248. MR1555394

[15] J. Serrin, On the interior regularity of weak solutions of the Navier-Stokes equations, Arch. Rational Mech. Anal. 9 (1962), 187-195. MR.0136885 (25:346)

[16] H. Sohr, The Navier-Stokes equations, An Elementary Functional Analytic Approach, Birkhäuser advanced texts, 2001. MR.1928881 (2004b:35265)

[17] H. Sohr and W. von Wahl, On the regularity of the pressure of weak solutions of Navier-Stokes equations, Arch. Math. 46 (1986), 428-439. MR847086 (87g:35190)

[18] M. Struwe, On partial regularity results for the Navier-Stokes equations, Comm. Pure Appl. Math. 41 (1988), 437-458. MR933230 (89h:35270)

[19] M. Struwe, On a Serrin-Type Regularity Criterion for the Navier-Stokes Equations in Terms of the Pressure, J. Math. Fluid Mech. 9 (2007), 235-242. MR.2329267(2008i:35185)

[20] Y. Zhou, A new regularity criterion for the Navier-Stokes equations in terms of the gradient of one velocity component, Methods Appl. Anal. 9 (2002), 563-578. MR2006605 (2004f:35141)

[21] Y. Zhou, Regularity criteria in terms of pressure for the 3-D Navier-Stokes equations in a generic domain, Math. Ann. 328 (2004), 173-192. MR2030374 (2004j:35229)

[22] Y. Zhou, A new regularity criterion for the Navier-Stokes equations in terms of the direction of vorticity, Monatsh. Math. 144 (2005), 251-257. MR2130277 (2006a:35243)

[23] Y. Zhou, A new regularity criterion for weak solutions to the Navier-Stokes equations. J. Math. Pures Appl. 84 (2005), 1496-1514. MR.2181458(2006g:35210)

[24] Y. Zhou, On regularity criteria in terms of pressure for the Navier-Stokes equations in $\mathbb{R}^{3}$, Proc. Amer. Math. Soc. 134 (2006), 149-156. MR.2170554(2006i:35288)

[25] Y. Zhou, On a regularity criterion in terms of the gradient of pressure for the Navier-Stokes equations in $\mathbb{R}^{n}$, Z. Angew. Math. Phys. 57 (2006), 384-392. MR2228171 (2007g:35179)

[26] Y. Zhou and S. Gala, Logarithmically improved regularity criteria for the Navier-Stokes equations in multiplier spaces, J. Math. Anal. Appl. 356 (2009), 498-501. MR2524284 